\title{
PARTICLE SIZE ANALYSIS AND FLOW OF POWDERS
}

$\mathrm{T}$ HE second regional meeting on "Particle Size Analysis" was held on October 2 at the University of Sheffield under the euspices of the Whiting and Industrial Powders Research Council. Like the first meeting at Hatfield, it attracted an audience of about 200 people from all parts of the country, and on this occasion one person from as far away as Norway. There was also an opportunity of visiting a specially arranged exhibition of particle size analysis equipment, at which sixteen exhibitors displayed twenty-four instruments. The day was divided into two sessions, particle size analysis being discussed in the morning and the flow of powders in the afternoon.

The chairman of the morning session was Mr. D. C. Soul, director of research of the Whiting and Industrial Powders Research Council. The first lecture, by Dr. B. H. Kaye, also of the Whiting and Industrial Powders Research Council, entitled "A Random Number Technique for Investigating the Accuracy of Microscope Methods of Particle Size Analysis", gave, as he himself said, the mathematically less-expert particle size analyst a 'do-it. yourself kit' for establishing the confidence limits to be placed in a given microscope particle count, or to compute the number of counts necessary to give a definite confidence. The method depends on assigning numbers corresponding to those in the size classes of particles found or anticipated, then to obtain random numbers from tables or otherwise, and interpret each number as a particle. Repetition of this process, involving no mathematics at all, allows a number of curves to be drawn, which will be enclosed by a 'scatterband' indicative of the purely statistical variations to be anticipated. but stripped of all experimental or operators' errors. The technique was illustrated by discussions of several typical analyses. A technique of 'stratified' sampling, in which the particles under test are divided into overlapping groups, which are counted separately and later recombined, was shown to give narrower confidence limits at no extra effort. The questions dealt with the need for preliminary counts of unknown samples, 'homogenizing' the results of the stratified groups to a unified whole, and the fact that the confidence limits of the whole, the average size, will be better than that of any particular group, which might be of interest, for example, for unwanted coarse material.

The second lecture on "The Use of the Ontical Microscope in Particle Size Analysis", by Dr. V. Timbrell, was read in his absence by three of his co-workers at the Pneumoconiosis Research Unit of the Medical Research Council and the Welsh School of Pharmacy. Mr. Reynolds described the electro-optical arrangements of the 'double image' microscope designed by Dr. Timbrell, in which two images of the same particle are produced by a vibrating mirror. The amplitude of vibration is adjusted until the images are just contiguous, when the particle diameter in the direction of vibration can be read from a calibrated meter. Mr. Cook discussed the advantages to be gained by this method, which lends itself to a certain degree of automation by comparison with the graticule method. Dr. Barnett amplified this theme and supported it with figures showing the vastly increased reproducibility in inter- and intra-operator comparisons, because the operator has only to find the position when two images precisely join, rather than estimate the sizes of irregular areas. Up to 400 particles can be counted in $10 \mathrm{~min}$. The questions were concerned with the price and availability of the microscone. the need or otherwise to make sure of random orientation of the particles. the possibility of using a projection method or examining photomicrographs rather than slides, and methods of dispersing certain particles for mounting on slides. Interesting answers to these latter questions suggested thixotropic liquids and, for completely three-dimensional random distribution, dispersing the particles in thermosetting resin and eventually preparing a section.

Dr. J. A. Kitchener, of the Royal School of Mines, took the chair for the afternoon session. During the first lecture, "The Flow of Powders", Dr. J. C. Williams, principal lecturer in powder technology at the Bradford Institute of Technology, gave a series of demonstrations to show the segregation of powders by size when being filled into a hopper. Fertilizer fractions differing $5: 1$ in diameter, the smaller particles being dyed green or black, segregated distinctly in the 'Perspex' containers and hoppers. The effect is caused by the fact that the smaller particles cannot flow down the slope of the heap, which is dotted with excessively large 'potholes'. Hoppers with more nearly vertical walls exhibit this phenomenon less. Similar demonstrations and slides showed the absence of flow at or near the hopper walls as they were emptied. This means that all except monodisperse powders will unmix when being charged into or discharged from a hopper. a phenomenon serious enough to merit close attention. The questions elicited a number of interesting facts - to name but one, that density and shape factors may also promote segregation, but in a conflict of factors, size is always predominant. Some desegregation becomes noticeable at particle diameter ratios $1: 1 \cdot 2$.

Mr. R. L. Brown, deputy director of the British Coal Utilization Research Association, discussed "Angles of Friction and Sliding of Non-Cohesive Powders". The angle of repose is the steepest angle against the horizontal of a heap of material at rest; the angle of sliding is the angle to the horizontal of the interface between stationary and moving material of a bed of powder discharging through an aperture. These angles depend not only on the material itself, but also on the geometry of the container and the manner of forming the heap; stress distributions are all-important, so that reproducible but mutually exclusive results may be obtained. Departure of the particles from sphericity and the presence of fines tend to increase the angles. In order to relate them to a more fundamental property, the angle of internal friction observed when a powder is sheared, is introduced; except against very rough surfaces, the angle of external friction is usually smaller. Internal friction and sliding as shown by the crater above the circular discharge aperture appear to be related. There are considerable differences in results derived from two- and three-dimensional surfaces, for example, in long, rectangular hoppers as against circular ones. Questions concerned the coating of powders, which may aid flow by destroying aggregates, different forms of 'bridging' in hoppers, and the advantages (starting a movement) and disadvantages (compaction) of vibrators in hoppers.

The last speaker of the day, Dr. L. Cohen, of Simon. Carves, Ltd., discussed "The Flow of Cohesive Bulk Solids". Rather than see how particle properties can be made to explain bulk properties, the lecturer described Prof. Jonike's approach of considering the heap as a homogenous, isotropic, plastic material developing strength under its own compacting weight, a conception that has also been found fruitful in soil mechanics. The principles of the Jenike shear cell, in which a compacted powder is sheared $(a)$ across its bulk and $(b)$ against a solid surface, were explained. The powder breaks down and begins to flow when its cohesive shear strength is exceeded. The practical history of the powder (dampness, tem. 
perature, residence in the hopper) is of crucial importance, but given the right conditions of flow function can be derived which is an invaluable aid to hopper design, and which is capable of forecasting differences in flow behaviour of otherwise seemingly identical powders. The questions dealt with the design of bunkers, the degree of standardization of Jenike's procedure and its applicability to damp and wet materials, the treatment of powders of very low bulk density (magnesium carbonate at $\left.8-9 \mathrm{lb} . / \mathrm{ft}^{3}\right)$, mechanical aids to hopper discharge and the effects of flow-promoters.

The meeting was closed with a vote of thanks to speakers, audience and organizers by Dr. Kitchener, who also announced that preparations for a third regional meeting on powder technology topics in the spring were under way.

\section{TRANSPORT AND TRAFFIC NOISE}

A SYMPOSIUM on "Transport and Traffic Noise" was held in the University of Southampton during September 27-28. The symposium, which was sponsored by the Society of Acoustic Technology and the Institute of Sound and Vibration Research of the University of Southampton, was attended by about 130 people representing various firms related to the motor industry, local government authorities, universities and colleges of advanced technology.

The symposium was opened with a lecture by Prof. E. J. Richards, the director of the Institute of Sound and Vibration, on the impact of the report of the Committee on the Problem of Noise, headed by Sir Alan Wilson. Prof. Richards, who was a member of the Committee, directed attention to the startling increase, which occurred between the 1948 and 1961 noise surveys, in the number of people who notice noise outside their homes42 per cent in 1948 and 91 per cent in 1961. The statistics given in the report suggest that road traffic noise is largely to blame for this incroase. In fact, of the various external noises, 36 per cent of people questioned in 1961 found road traffic noise disturbing, compared with 9 per cent who were disturbed by aircraft noise. Prof. Richards discussed the findings of the Wilson Committee in general terms and described some of the recommendations which it had made.

The second lecture was given by Dr. B. W. Robinson (National Physical Laboratory) on the measurement of noise. Dr. Robinson outlined the various units which have been introduced for the measurement of noise and discussed the relation between the objective (db), the frnquency weighted decibel (dbA), the unit of subjective assessment (phon), and the attempts which had been made by Stevens, Zwicker and others, to compute a single loudness figure which agrees closely with the phon. Dr. Robinson also discussed the standard practices which have been suggested for rating the noisiness of motor vehicles and which may soon be implemented by law.

The afternoon session was devoted to two technical lectures, by Dr. T. Priede (C.A.V., Ltd., Warple Way, London, W.3) and Dr. P. O. A. L. Daries (Institute of Sound and Vibration Research). Dr. Priede discussed the subject of engine noise, with particular reference to Diesel engine noise, illustrating his talk not only with slides, but also with tape recordings, which proved to be a much more efficient means of showing the result of decreasing a noise by a fow decibels. Dr. Priede discussed the mechanics by which noise was created in a Diesel engine and described some of the extensive investigations which have bean carried out at C.A.V., Ltd.. to reduce it. Special mention was made of air-intake nnise, the reduction in noise which may be ohtrined by smoothing the cylinder pressure, and the noise produced by resonance of the engine casing.

In his lecture on silencer design. Dr. Davies compared the results which may be obtained from the traditional acoustic thaory of silencers, and the new shock wave theory which has been investigated by him and his co-workers at Southampton. Single-pulse shock wave theory can be used in discussing an exhaust system, because for a typical four-stroke engine running at 3,000 r.p.m. the primary pulses travel down the exhaust pipe at intervals of about $18 \mathrm{ft}$. Dr. Davies showed that the single pulse theory may be used to give a picture of the gas flow through the exhaust system, to calculate the back pressure, and to determine the attenuation which will be produced by the system. The acoustic filter theory still has its place in the field of harmonic response, but the single pulse theory describes the transient response in a way the older theory could never do.

The evening session of the conference consisted of an open lecture by Mr. H. Purkis, of the Building Research Station, on the subject of transport noise and town planning. Mr. Purkis first directed attention to the way in which noise nuisance has been growing and is likely to grow in the future, owing to the tremendous increase in vehicle ownership and industrialization. He then discussed some of the proposals which had been suggested for combating the nuisance in new towns. Motor-vehicles could not be prohibited from a town without killing all commerce, and it could not be expected that the noise-lovels which were to be laid down for cars would be able to counteract the increase in overall noise-level which would be produced by the increasing number of vehicles.

Since overall noise-level must be expected to increase, means must be found to prevent the noise reaching houses, shops and offices. Mr. Purkis discussed sunken roads, smooth flowing motor-ways lined with specially insulated buildings to prevent the noise permeating to other dwellings, as well as some of the unsuccessful (that is, noise-producing) devices which were being used by traffic engineers to-day-one-way streets, which lead to faster, more noisy, traffic, and the use of side streets, which distributes the noise to parts of the town which were previously quiet.

On the Saturday morning, consideration was given to the problem of noise as it affects the passengers in a car. The first lecturer was Dr. G. M. L. Gladwell (Institute of Sound and Vibration Research), who presented a review of the literature on the subject. $\mathrm{He}$ paid particular attention to the noise caused by the passage of the tyres over the road, to the way in which this was transmitted through the car structure, and to the mechanical/acoustic properties of the passenger cabin. He also stressed the need for more fundamental investigation into the vibration characteristics of a car, and the use of electronic analogue computers to make the investigation of the vibration characteristics of a projected design more logical and less a matter of guesswork. In the extended riscussion period following this review, representatives from Peugeot, Ford, Humber, Pirelli and Dunlop contributed from their own experience. The discussion centred on the road noise problem. M. J. Genestier, director of the Peugeot Research Laboratory, explained how they had devised the suspension system of their 404 model sprcifically for the Michelin $X$ tyre, a tyre which excites vibration in a frequency range much narrower than others. and had managed so to tune the suspension system that the vibrations that were excited were not transmitted to the car. Other representatives stressed the need for car and tyre manufacturers to co-operate, and consider when designing 8 car the characteristics of the tyres which were to be fitted. 